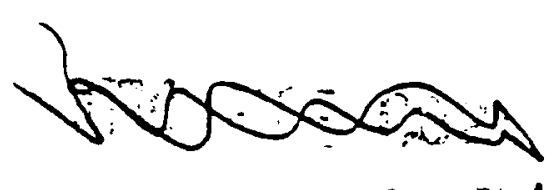

HengR.TR. \& $9 \cdot 0 \div \div$

Wavefront Propagation for Reaction-Diffusion

Systems of PDE

Q

co

$\infty$

$\begin{array}{ll} & \\ 1 & \end{array}$

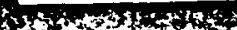

ton

ind 7

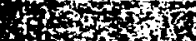

ts

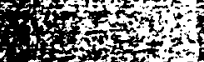

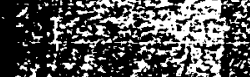

3its

\%

tis

tet

$\Rightarrow$

is

t.

to

xite

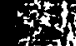

sits

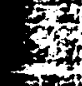

$2 x$

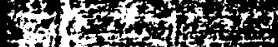

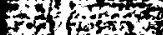

(1)

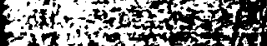

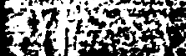

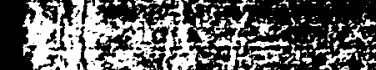

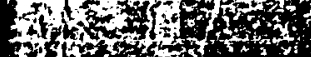

ats 78

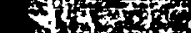

1 3

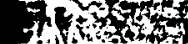

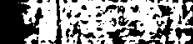

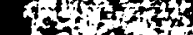

क

\section{DISTR'BUTION ST ATEMENT $A$ \\ Apprezed for public rolecen;}

Distribution Unlimited

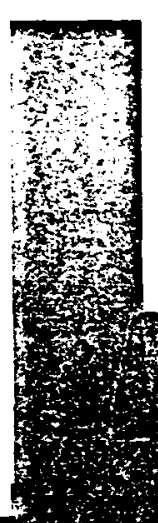

G. Barles, L.C. Evans and P.E. Souganidis

Xialch $: 930$

LCDS $\# 5 !-3$

Eefschetzenter for Bynamicalisystems $\operatorname{and}$

Center for Control Sciences

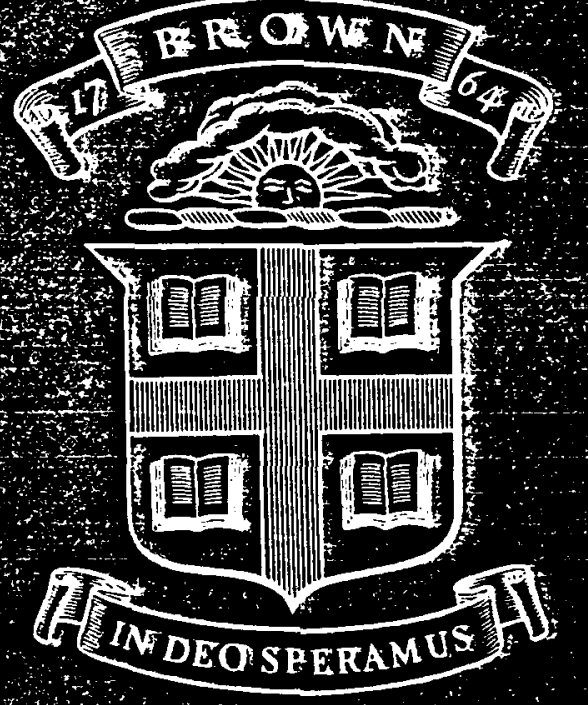

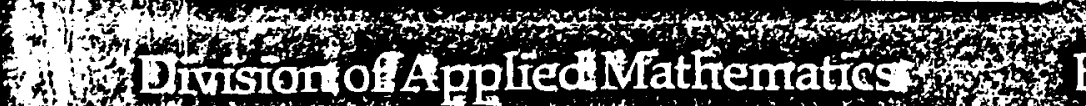

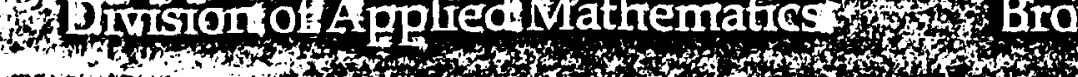

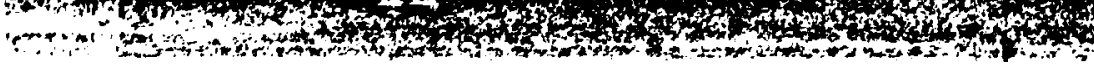

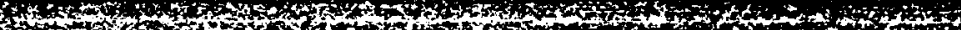

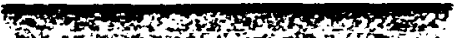

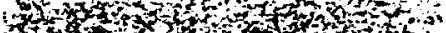

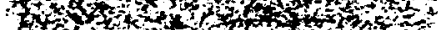

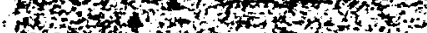

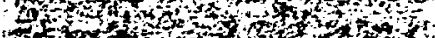

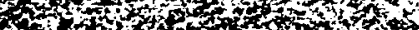

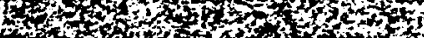

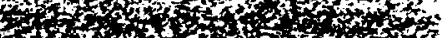
37. t5
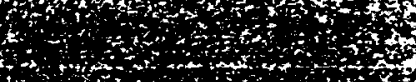


\section{Wavefront Propagation for Reaction-Diffusion} Sy'stems of PDE

\section{by}

G. Barles, L.C. Evans and P.E. Souganidis

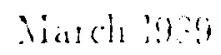

LCDS $\#-3$

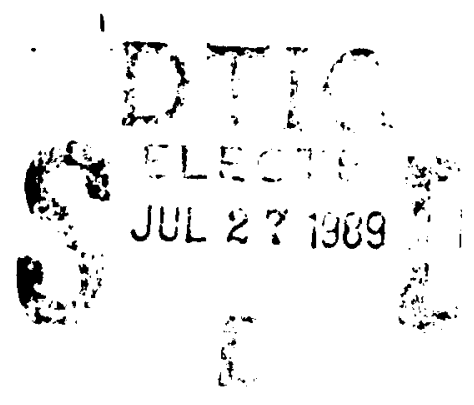




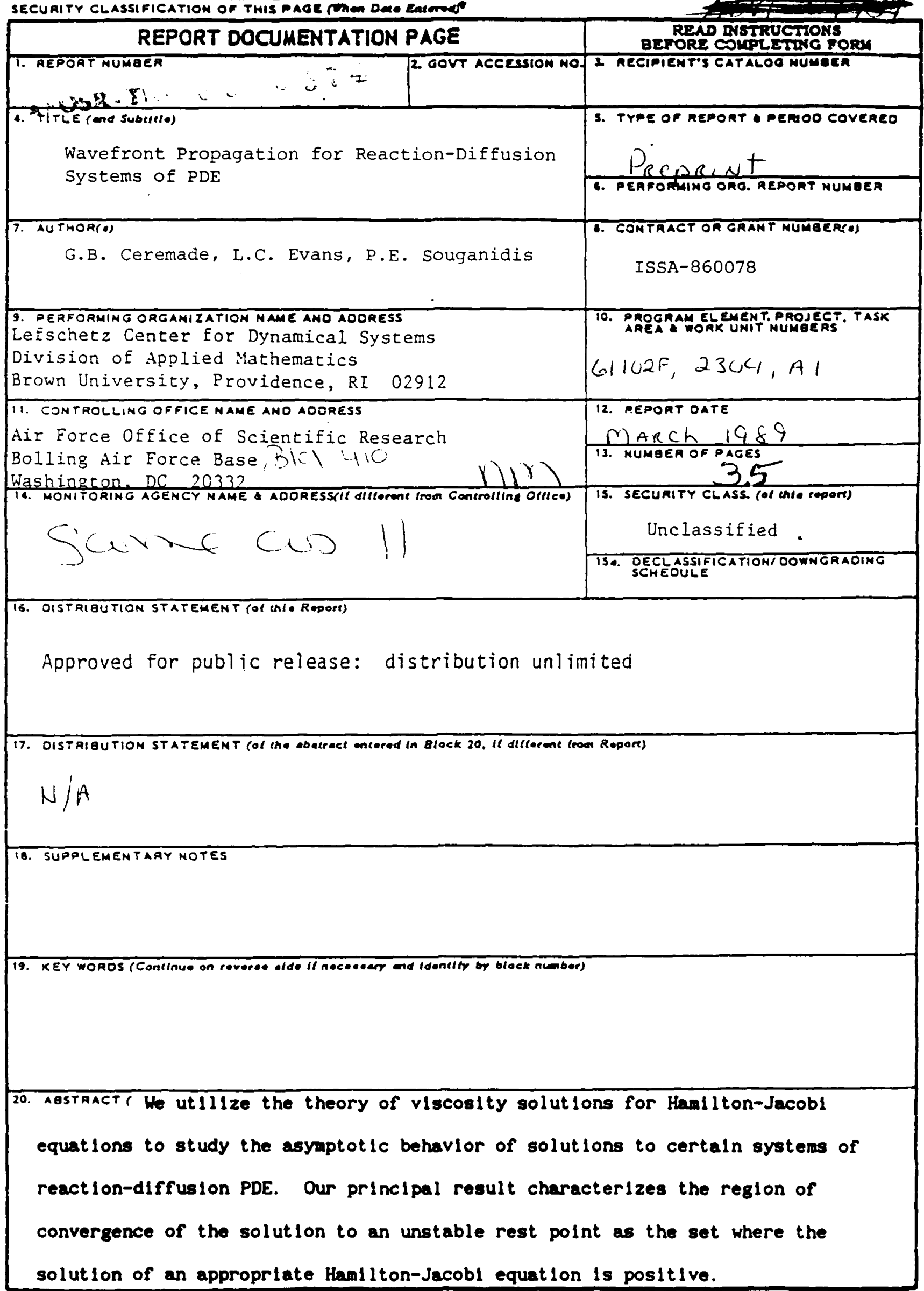

DO I JAMM 1473 EDITION OF I MOV AS IS OESOLETE

S/N 0102- U. O14.6601
UNCLASSIFIED

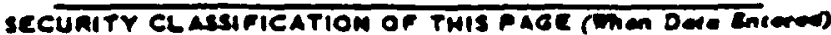




\title{
Wavefront Propagation for Reaction-Diffusion \\ Systems of PDE
}

by

\section{G. Barles}

CEREMADE

Universite Paris IX-Dauphine

75775 Paris Cedex 16, France

L.C. Evans"

Department of Mathematics

University of Maryland

College Park, MD 20742 USA
P.E. Souganidis

Division of Applied Mathematics

Brown University

Providence, RI 02912 USA

\begin{abstract}
\1
Abstract We utlilize the theory of viscosity solutions for Hamilton-Jacobl equations to study the asymptotic behavior of solutions to certain systems of reaction-diffusion PDE. Our principal result characterizes the region of convergence of the solution to an unstable rest point as the set where the solution of an appropriate Hamilton-Jacobl equation is positive.

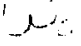

- Supported in part by the NSF under grant DMS-86-01532

- Supported in part by the NSF under grants DMS-8601258 and DMS-8657464, by the AFOSR under agreement AFOSR-ISSA-860078, by the ONR under contract N0014-83-K-0542 and by the ARO under contract DAALO3-86-K-0074.
\end{abstract}


M. I. Freidlin ([9], [10]) has recently introduced probabilistic techniques into the study "wavefront propagation" for systems of reactiondiffusion PDE. The motivating idea is that should a reaction-diffusion system possess only a single unstable and a single stable equilibrium, then the solution $u$ of the system will presumably tend to "switch" for large times from near the former to near the latter state. A mathematical problem is then to describe this transition, ideally in terms of simpler quantitles than those governing the full, detalled behavior of the entire system of PDE. More precisely, to study the reaction-diffusion problem for large times of order $\varepsilon^{-1}$, Freidlin suggests a $\varepsilon^{-1}$ rescaling in the space and time variables; so that our attention turns to the solutions $u^{\varepsilon}$ of certain $\varepsilon$-dependent systems of PDE. We then hope to show that as $\varepsilon \rightarrow 0$, the functions $u^{\varepsilon}$ converge in some region $G \subset \mathbb{R}^{n} \times(0, \infty)$ to the stable point, and in the opposite region $\left[\mathbb{R}^{n} \times[0, \infty)\right] \backslash G$ to the unstable point. We simultaneously hope to describe geometrically or analytically this set G, whose boundary we envision as a spreading wavefront separating regions with quite different 1 imiting behavior.

This paper, which is an extension to systems of earller work [6] on single equations, brings to bear purely PDE techniques to this problem, especially the theory of viscosity solutions on Hamliton-Jacobl equations, due to Crandall-Lions [3]. The connection with the foregoing discussion is that, the region $G$ alluded to above is the set where the solution $J$ of a certain Hamliton-Jacobi equation is negative. Our procedure for understanding the limiting behavior of the solution of the reaction-diffusion system of PDE is thus first of all to bulld an approprlate Hamlitonian $H$ out of the data given in the problem, second to solve the resulting Hamilton-Jacobl equation for $J$, and last to demonstrate the different $11 \mathrm{miting}$ behavior of the solutions $u^{\varepsilon}$ of the scaled system on the sets $\{J<0\}$ and $\{J>0\}$. We 
Informally regard the Hamlitonlan as controlling somehow the rate of instabllity of the unstable point. We are thus able to characterize the asymptotic behavior of the "complicated" reaction-diffusion system in terms of the "simple" Hamilton-Jacobl equation. Thls possiblilty, flrst ldentifled by Freidin [9] in rather different terms, is attractive, but of course requires for this implementation many structural assumptions on the nonlinearities, which we list below. It would of course be quite interesting to extend our results, or at least the point of view espoused above, to systems with more general nonlinearities.

More precisely now, we intend to Investigate the scaled reactiondiffusion system:

$(1.1)_{\varepsilon}$

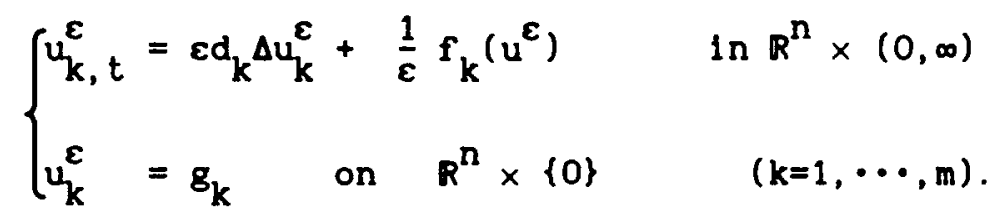

Here the constants $d_{k}(1 \leq k \leq m)$, and the functions

$$
\text { g: } \mathbb{R}^{n} \rightarrow \mathbb{R}^{m} \text { and } f: \mathbb{R}^{m} \rightarrow \mathbb{R}^{m}
$$

are given, where we write $g=\left(g_{1}, \cdots, g_{m}\right), f=\left(f_{1}, \cdots, f_{m}\right)$. The unknown is $u^{\varepsilon} \equiv\left(u_{1}^{\varepsilon}, \cdots, u_{m}^{\varepsilon}\right)$. We will assume that

$$
d_{k}>0 \quad(k=1, \cdots, m),
$$

and that the functions $g, f$ are smooth, bounded and Lipschitz. In addition we suppose that

$$
\mathbf{g}_{k} \geq 0 \quad(k=1, \cdots, m)
$$

and

$$
G_{0}=\left\{g_{k}>0\right\}
$$$$
(k=1, \cdots, m)
$$ 
is a bounded, smooth subset of $R^{n}$. Under these assumptions there exists a unique smooth solution $u^{\varepsilon}$ of the PDE (1.1) with

$$
u_{k}^{\varepsilon}>0 \quad \text { in } R^{n} \times(0, \infty) \quad(k=1, \cdots, m)
$$

Our essential assumptions all concern the reaction term $f$. First of all we suppose

$$
f(0)=0 \text {; }
$$

and also

$$
\left\{\begin{array}{l}
f_{k}\left(u_{1}, \cdots, u_{k-1}, 0, u_{k+1}, \cdots, u_{m}\right)>0 \text { if } \\
u_{1}, \cdots, u_{k-1}, u_{k+1}, \cdots, u_{m} \geq 0 \text { and } u_{1}>0 \text { for some Index } 1 \neq k
\end{array}\right.
$$

Consequently the vector vield f points strictly inward along the boundary of the positivity set

$$
\Pi \equiv\left\{u \in R^{m} \mid u_{1}>0, \cdots, u_{m}>0\right\},
$$

except at the point 0 , which is an equilibrium point for the system (1.1) $\varepsilon$. To ensure that our solutions $u^{\varepsilon}$ do not become unbounded as $\varepsilon \rightarrow 0$, we further hypothesize that

$$
\begin{aligned}
& \text { there exists a constant } \Lambda \text { such that } \\
& \qquad f_{k}(u) \leq 0 \\
& \text { if } u \in \Pi \text { and } u_{k} \geq \Lambda .
\end{aligned}
$$

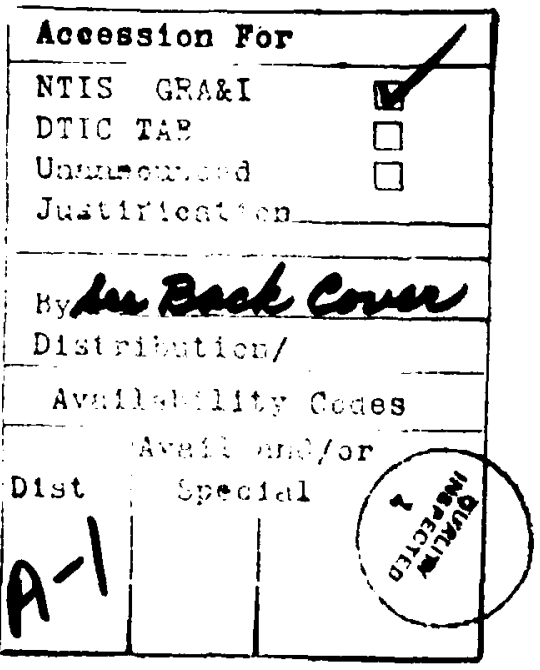




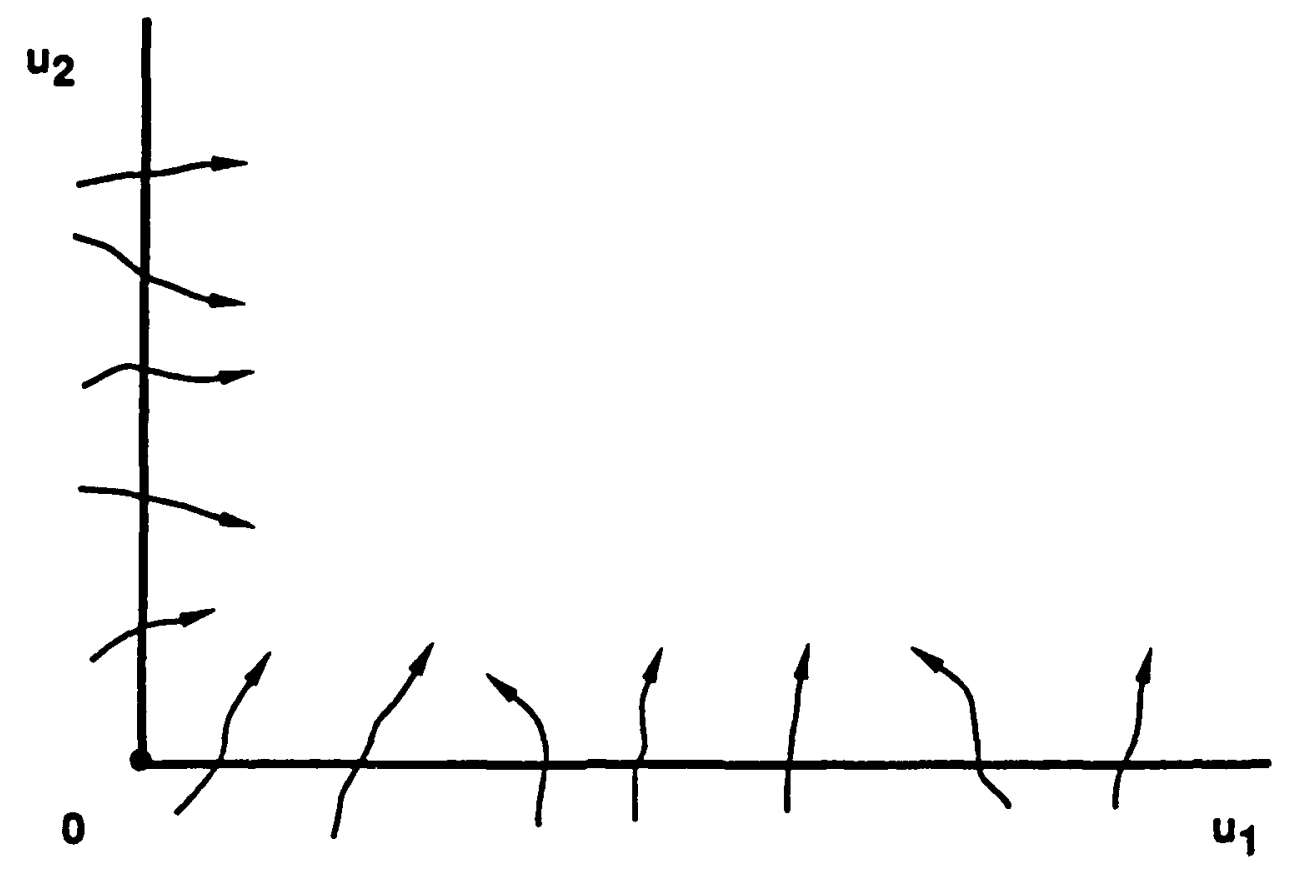

Figure 1

Next we set forth additional hypotheses which imply that the rest polnt 0 is unstabie. Let us define the $m \times m$ matrix

$$
C=\operatorname{Dr}(0)
$$

Df denoting the gradient of $f$. Thus

$$
c_{k 1}=f_{k, u_{1}}(0) \quad(1 \leq k, 1 \leq m)
$$

We assume

(F4)

$$
c_{k l}>0
$$

and

$$
f_{k}(u) \leq c_{k l} u_{1} \quad(u \in \Pi, k=1, \cdots, m) \text {, }
$$

where we employ the standard summation convention. 
for systems of the Kolmogorov-Petrovskil-Piskunov nonlinearity, discussed in $[6]$.

Our main result, Theorem 1, asserts that under hypotheses (F1) - (F5) $u^{\varepsilon}(x, t)$ converges as $\varepsilon \rightarrow 0$ to zero or not depending on whether $J(x, t)>0$ or $J(x, t)<0$, the function $J$ satisfying a Hamilton-Jacobl PDE whose structure we now describe. Given $p \in R^{n}$, define the $m \times m$ matrix

$$
B(p)=\operatorname{dlag}\left(\cdots, d_{k}|p|^{2}, \cdots\right)
$$

and then set

$$
A(p)=B(p)+C .
$$

Now the matrix $A(p)$ has positive entries, and so Perron-Frobenius theory asserts that $A(p)$ possesses a simple, real elgenvalue $\lambda^{0}=\lambda^{0}(A(p))$ satisfying

$$
\operatorname{Re} \lambda<\lambda^{0}
$$

for all other elgenvalues $\lambda$ of $A(p)$. Let us define then the Hamiltonian

$$
H(p)=\lambda^{0}(A(p)) \quad\left(p \in \mathbb{R}^{n}\right)
$$

See, for instance, [7] for a revlew of the varlous properties of $H$, and in particular a proof that $H$ is convex. We additionally set

$$
L(q)=\sup _{p \in R^{m}}(p \cdot q-H(p))
$$

$L$ is the Lasrangian assoclated with H. Finally we define for each point $(x, t) \in R^{n} \times(0, \infty)$ the action function

$$
J(x, t) \equiv \operatorname{lnf}\left\{\int_{0}^{t} L(\dot{z}(s)) d s \mid z(0) \in G_{0}, z(t)=x\right\}
$$

the infimum taken over all absolutely continuous functions

$$
z:[0, t] \rightarrow R^{n}
$$


satisfying the stated initlal and terminal conditlons. As we will see, J turns out to be the (unlque) solution of the Hamilton-Jacobl equation

$$
\begin{cases}J_{t}+H(D J)=0 & \text { in } R^{n} \times(0, \infty) \\ J=0 & \text { on } G_{0} \times\{0\} \\ J=+\infty & \text { on } \operatorname{lnt}\left(R^{n}-G_{0}\right) \times\{0\}\end{cases}
$$

in the viscosity sense (cf. Crandall-Lions (3), Crandall-Evans-Lions [2], etc.).

Theorem 1. Under hypotheses (F1)-(F5) we have

$$
\lim _{\varepsilon \rightarrow 0} u^{\varepsilon}=0
$$

unlformly on compact subset of $\{\mathrm{J}>0\}$

and

(1.10) $\quad \lim \operatorname{linf}_{\varepsilon \rightarrow 0} u_{k}^{\varepsilon}>0 \quad(k=1, \cdots, m)$ uniformly on compact subset of $\{J<0\}$.

We loosely interpret this theorem as describing how the Hamlltonian $H$, which depends upon both $c=D f(0)$ and the diffusion constants $d_{1}, \cdots, d_{m}$, controls the instability of the equilibrium point $u=0$.

Remark We should note also that it is possible to refine conclusion (1.10) by making further assumptions on the behavior of the vector fleld $f$ in $\Pi$. As in Freidlin [9], we may for instance assume that there exists a unique equilibrium point $a \in \pi$ which is asymptotically stable for the flow generated by the vector fleld $f$, as in the following lllustration. 


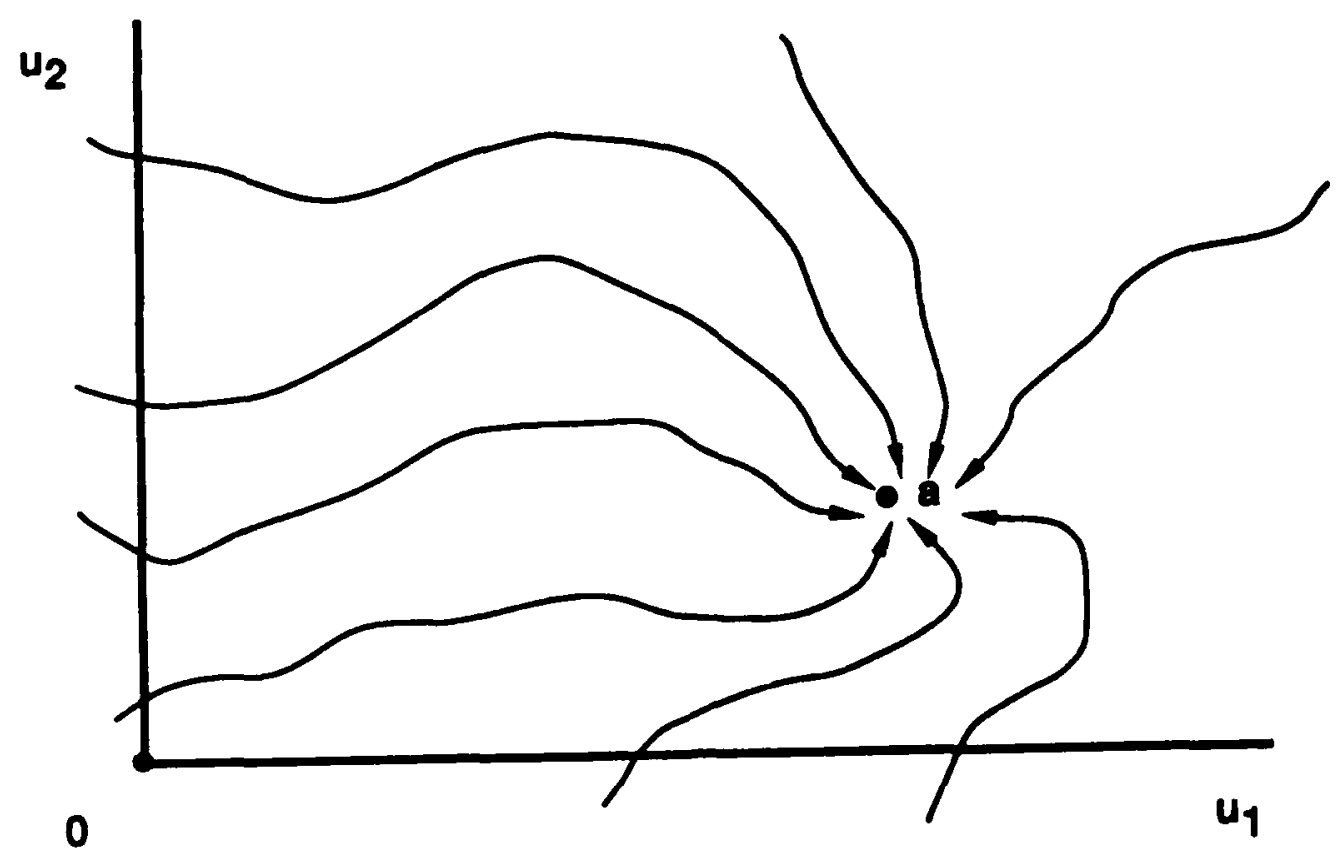

Figure 2

Under varlous fairly stringent technical assumptions, it is then possible to show that

$$
\lim _{\varepsilon \rightarrow 0} u^{\varepsilon}=a \quad \text { uniformly on compact subsets of }\{J<0\} \text {. }
$$

We may consequently thlnk of the boundary of the set $G \equiv\{J<0\}$ as a "propagating wavefront" in the sense explained above.

Assertions (1.9), (1.11) are attractive, but the sufficlent conditions we know to improve (1.10) to (1.11) are rather unsatisfactory technically. (For instance, we could assume that the diffusions constants are all equal and that $f$ has a convex Lyapunov function assoclated with the stable point $a$, etc.). For this reason, we will not here formulate any precise assertions leading to (1.11), but instead refer the reader to Freldlin [9], [10] for more information on this polnt. a

Our paper is organized so that the proof of Theorem 1 appears in \$3, \$2 belng devoted to some preliminary estimates. The main idea, following [8], 
is to perform a change of varlables, after which we send $\varepsilon \rightarrow 0$. This approach Is greatly complicated for the present case of systems, since we lose the maximum principic and consequently many of the estimates avallable in [6] for the scalar case. We depend instead upon some recent techniques introduced by Ish11 [11] and Barles-Perthame [1] in Hamilton-Jacobl theory, whlch allow us to proceed with only supremum-norm bounds. This is a great advantage since better estimates seem to be unobtalnable, but the price is a far greater Intricacy in some of the convergence proofs. We wlll encounter for instance troubles in interpreting in just what sense certain solutions take on their Initial values. An appendix (\$4) develops some useful theory on such questions, which we will need in $\$ 3$.

Finally we let us note that although Freldlin's work has greatly inspired us, we belleve that the PDE technlques developed here (and in [6]) provide Information which is not at all clear from the probabllistlc viewpoint. We for instance do not require the fairly specific structural assumptions on the nonlinearity $f$ utllized in $[9, p .467]$. 


\section{Preliminary est Imates}

Henceforth we always suppose hypotheses (F1)-(F5) to be in effect.

Lemma 2.1 There exists a constant $C_{1}$ such that

$$
0<u_{k}^{\varepsilon} \leq c_{1} \quad-\ln R^{n} \times(0, \infty)
$$

for $k=1, \cdots, m$ and each $\varepsilon>q^{\prime}$.

Proof. Choose a smooth, bounded, Lipschitz vector fleld

$$
\text { 全: } \mathbb{R}^{\mathrm{m}} \rightarrow \mathbb{R}^{\mathrm{m}}
$$

such that

$$
\begin{cases}\hat{f}(u)=f(u) & (u \in \bar{\Pi}) \\ \text { and } & \\ & \hat{f}_{k}(u) \geq 0 \quad \text { if } u_{k}<0, k=1, \cdots, m ;\end{cases}
$$

this is possible in light of (F2). Now let $\hat{u}^{\varepsilon}$ be the unique, smooth solution of the system

(2.3)

$$
\begin{cases}\hat{u}_{k, t}^{\varepsilon}=\varepsilon d_{k} \Delta \hat{u}_{k}^{\varepsilon}+\frac{1}{\varepsilon} \hat{f}_{k}\left(\hat{u}^{\varepsilon}\right) & \text { in } R^{n} \times(0, \infty) \\ \hat{u}_{k}^{\varepsilon}=g_{k} & \text { on } R^{n} \times\{0\} .\end{cases}
$$

Choose

$$
\boldsymbol{n}: \mathbb{R} \rightarrow \mathbb{R}
$$

to be smooth and convex, w1th

(2.4)

$$
\begin{cases}\eta=0 & \text { on }(0, \infty) \\ \eta>0 & \text { on }(-\infty, 0)\end{cases}
$$

Then for each $k=1, \cdots$,

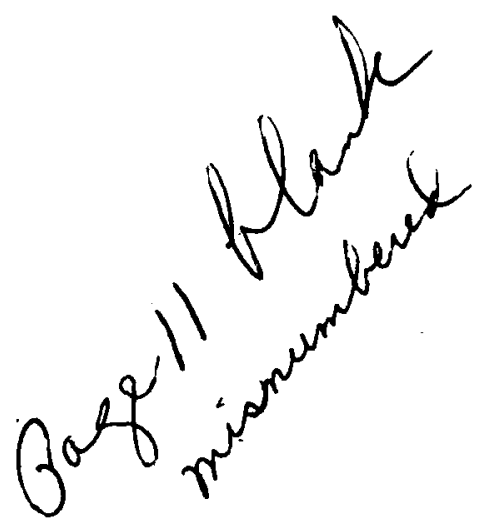




$$
\begin{aligned}
\frac{d}{d t} \int_{R^{n}} \eta\left[u_{k}^{\varepsilon}\right) d t & =-\varepsilon \int_{R^{n}} d_{k} \eta^{\mu}\left[u_{k}^{\varepsilon}\right]\left|D u_{k}^{\varepsilon}\right|^{2} d x \\
& =\frac{1}{\varepsilon} \int_{\mathbb{R}^{n}} \eta^{\prime}\left[u_{k}^{\varepsilon}\right] f_{k}\left(u^{\varepsilon}\right) d x \\
& \leq 0, \quad \text { by }(F 3) .
\end{aligned}
$$

Since

$$
\int_{R^{n}}-\left(\overline{\varepsilon_{k}}\right) d x=0,
$$

we discover

$$
u_{k}^{\varepsilon} \leq \theta \quad \text { in } R^{n} \times[0, \infty)
$$

3. Observe now that hypotheses (F1), (F2) and (F4) imply $f_{k}(u) \geq 0$ if $u_{k}^{\varepsilon} \geq 0$ is small enough $(k=1, \cdots, m)$. Thus the strong maximum princlple for parabolic equations $1 \mathrm{mplies}$

$$
u_{k}^{\varepsilon}>0 \quad \text { in } R^{n} \times(0, \infty), \quad(k=1, \cdots, m) \quad 0
$$

We next introduce, following [5], [6], [7], [8], etc. the new functions

$$
v_{k}^{\varepsilon} \equiv-\varepsilon \log u_{k}^{\varepsilon} \quad \text { in } \mathbb{R}^{n} \times(0, \infty) \quad(k=1, \cdots, m)
$$

A calculation shows that $v^{\varepsilon}=\left(v_{1}^{\varepsilon}, \cdots, v_{m}^{\varepsilon}\right)$ satisfles the system

$$
\left\{\begin{array}{l}
v_{k, t}^{\varepsilon}-\varepsilon d_{k} \Delta v_{k}^{\varepsilon}+d_{k}\left|D v_{k}^{\varepsilon}\right|^{2}=-\frac{f_{k}\left(u^{\varepsilon}\right)}{u_{k}^{\varepsilon}} \text { in } R^{n} \times(0, \infty) \\
v_{k}^{\varepsilon}=-\varepsilon \log g_{k} \text { on } G_{0} \times\{0\} \\
v_{k}^{\varepsilon}+\infty \quad \text { on } \operatorname{lnt}\left(R^{n}-G_{0}\right) \times(0\} .
\end{array}\right.
$$

Note also that in view of Lemma 2.1 we have

$$
v_{k}^{\varepsilon} \geq-\varepsilon \log C_{1} \quad \text { in } R^{n} \times(0, \infty)
$$


for $k=1, \cdots, m$ and each $\varepsilon>0$.

Lemma 2.2 For each compact subset $Q \subset C\left(R^{n} \times(0, \infty)\right) \cup\left(C_{0} \times(0, \infty)\right)$ there exists a constant $C_{2}(Q)$ such that

$$
\left|v_{k}^{\varepsilon}\right| \leq C_{2}(Q) \quad \text { in } Q
$$

for $k=1, \cdots, m$ and each $\varepsilon>0$.

Proof 1. Let 0 denote any open subset of $R^{n} \times(0, \infty)$. Suppose that $\phi$ is a smooth function satisfying

$$
\phi_{t}-\varepsilon d_{k} \Delta \phi+d_{k}|D \phi|^{2} \geq \lambda \quad \text { in } 0
$$

where $\lambda>0$ is a positive constant to be selected later. Assume now that $v_{k}^{\varepsilon}-\phi$ has a maximum at some point $\left(x_{0}, t_{0}\right) \in 0$. Then at the point $\left(x_{0}, t_{0}\right)$ we have

$$
\begin{aligned}
0 & \leq\left[u_{k}^{\varepsilon}-\phi\right]_{t}-\varepsilon d_{k} \Delta\left[v_{k}^{\varepsilon}-\phi\right] \\
& \leq-\frac{f_{k}\left(u^{\varepsilon}\right)}{u_{k}^{\varepsilon}}-\lambda \quad \text { by }(2.6)_{\varepsilon} \text { and }(2.8) .
\end{aligned}
$$

But

$$
\begin{aligned}
-f_{k}\left(u^{\varepsilon}\right) & =-f_{k}\left(\cdots, u_{k-1}^{\varepsilon}, 0, u_{k+1}^{\varepsilon}, \cdots\right) \\
& +\left[f_{k}\left(\cdots, u_{k-1}^{\varepsilon}, 0, u_{k+1}^{\varepsilon}, \cdots\right)-f_{k}\left(\cdots, u_{k-1}^{\varepsilon}, u_{k}^{\varepsilon}, u_{k+1}^{\varepsilon}, \cdots\right)\right] \\
& \leq c u_{k}^{\varepsilon}, \text { according to Lemma } 2.1 \text { and }(F 2) .
\end{aligned}
$$

Substituting above we discover

$$
0 \leq \mathrm{C}-\lambda \text {, }
$$


a contradiction for $\lambda$ large enough.

2. We may assume that for some $r>0$ the ball $B(0, r)$ lies in $G_{0}$. Set then

$$
\phi^{2}=\frac{1}{r^{2}-|x|^{2}}+\beta t+\gamma \quad(|x|<r, t \geq 0)
$$

$\beta$ and $\gamma$ picked as indicated below (cf. [5], [7]). We compute

$$
\begin{gathered}
\phi_{t}^{1}-\varepsilon d_{k} \Delta \phi^{1}+d_{k}\left|D \phi^{1}\right|^{2} \\
=\beta-\varepsilon d_{k}\left[\frac{2 n}{r^{2}-|x|^{2}}+\frac{8|x|^{2}}{\left(r^{2}-|x|^{2}\right)^{3}}\right]+d_{k} \frac{4|x|^{2}}{\left(r^{2}-|x|^{2}\right)^{4}} \\
\geq \lambda \quad \text { in } B(0, r) \times(0, \infty) \quad(k=1, \cdots, m),
\end{gathered}
$$

provided $\beta>0$ is large enough. If we then adjust $\gamma$ to be so large that

$$
\phi^{1} \geq v_{k}^{\varepsilon} \quad \text { on } B(0, r) \times\{0\} \text {. }
$$

we deduce from Step 1 that

$$
v_{k}^{\varepsilon} \leq \phi^{1} \quad \text { in } B(0, r) \times(0, \infty) .
$$

Consequent ly

$$
v_{k}^{\varepsilon} \leq C \quad \text { in } B(0, r / 2) \times(0, T) .
$$

for $k=1, \cdots, m$ and each $\varepsilon>0, \quad T>0$.

3. Now write

$$
\phi^{2}=\frac{\alpha|x|^{2}}{t}+\beta t+\gamma \quad\left(|x|>\frac{r}{2}, t>0\right)
$$

We calculate

$$
\phi_{t}^{2}-\varepsilon d_{k} \Delta \phi^{2}+d_{k}\left|D \phi^{2}\right|^{2}=-\frac{\alpha|x|^{2}}{t}+\beta-\varepsilon d_{k} \frac{2 n}{t}+d_{k} \frac{4 \alpha^{2}|x|^{2}}{t^{2}}
$$




$$
2 \lambda \text { in }\left(R^{n}-B(0, r / 2)\right) \times(0, \infty) \text {. }
$$

provided $\alpha, \beta>0$ and large enough. We now pick $\gamma$ large enough to ensure

$$
\phi^{2} \geq v_{k}^{\varepsilon} \quad \text { on } A B\left(0, r^{\prime} 2\right) \times(0, T) \text {. }
$$

This is possible owing to estimate (2.9). Consequently Step 1 implies

$$
v_{k}^{\varepsilon} \leq \phi^{2} \quad \ln \left(R^{n}-B(0, r / 2)\right) \times(0, T)
$$

for $k=1, \cdots, m$ and each $\varepsilon>0, T>0$.

This bound and (2.9) lead at once to the estimate stated in the Lemma.

$$
\text { 口 }
$$


3. Proof of Theorem 1.

For each $(x, t) \in R^{n} \times(0, \infty)$ we set

$$
v_{k *}(x, t)=\lim _{\substack{\varepsilon \rightarrow 0 \\(y, s) \rightarrow(x, t)}} v_{k}^{\varepsilon}(y, s) \quad(k=1, \cdots, m)
$$

and

$$
v_{k}^{*}(x, t)=\lim _{\substack{\varepsilon \rightarrow 0 \\(y, s) \rightarrow(x, t)}} v_{k}^{\varepsilon}(y, s) \quad(k=1, \cdots, m)
$$

We additionally write

$$
v_{*}=\min _{1 \leq k \leq m} v_{k^{*}}, v^{*}=\max _{1 \leq k \leq m} v_{k}^{*}
$$

We intend to show that $v_{*}$ is a lower semicontinuous supersolution, and $v$ is a upper semicontinuous subsolution, of an appropriate variational inequality involving the Hamiltonian $\mathrm{H}$ defined in $\$ 1$.

Proposition 3.1. We have

$$
\min \left(v_{*}+H\left(D v_{*}\right), v_{*}\right) \geq 0 \quad \text { in } \mathbb{R}^{n} \times(0, \infty)
$$

in the viscosity sense, and

$$
v_{*}=\left\{\begin{array}{cc}
0 & \text { on } G_{0} \times\{0\} \\
+\infty & \text { on }\left(R^{n}-\bar{G}_{0}\right) \times\{0\} .
\end{array}\right.
$$

Proof 1. Because of (2.7) it is clear that

$$
v . \geq 0
$$

To demonstrate that

$$
v_{* t}+H\left(D v_{*}\right) \geq 0 \quad \text { in } R^{n} \times(0, \infty)
$$


in the viscosity sense, we $f 1 x$ a smooth test function $\phi$ and assume

(3.6) $\left\{\begin{array}{l}v_{0}-\phi \text { has a strict local minimum at some point } \\ \left(x_{0}, t_{0}\right) \in \mathbb{R}^{n} \times(0, \infty) .\end{array}\right.$

We must prove

$$
\phi_{t}+H(D \phi) \geq 0 \text { at }\left(x_{0}, t_{0}\right) \text {. }
$$

2. Let $\left(\psi_{1}, \cdots, \psi_{k}\right)$ be a positive eigenvector of the matrix $A\left(D \phi\left(x_{0}, t_{0}\right)\right)$ corresponding to the principal eigenvalue $\lambda^{0}\left(A\left(D \phi\left(x_{0}, t_{0}\right)\right)=H\left(D \phi\left(x_{0}, t_{0}\right)\right)\right.$.

Note then

$$
v_{*}(x, t)=\lim _{\substack{\varepsilon \rightarrow 0 \\(y, s) \rightarrow(x, t)}} \min _{1 \leq 1 \leq m}\left[v_{1}^{\varepsilon}(y, s)+\varepsilon \log \psi_{1}\right] .
$$

Combining (3.6) and (3.8), we deduce that there exists an index $k \in\{1, \cdots, m\}$, a sequence $\varepsilon_{r} \rightarrow 0$, and points $\left(x^{\varepsilon_{r}}, t^{\varepsilon_{r}}\right)$ such that

(3.9) $v_{k}^{\varepsilon_{r}}\left[x^{\varepsilon_{r}}, t \varepsilon_{r}\right]+\varepsilon_{r} \log \psi_{k}=\min _{1 \leq 1 \leq m}\left[v_{1}^{\varepsilon_{r}}\left[x^{\varepsilon_{r}}, t^{\varepsilon_{r}}\right]+\varepsilon_{r} \log \psi_{1}\right] \rightarrow v_{*}\left(x_{0}, t_{0}\right)$,

(3.10) $\quad\left[v_{k}^{\varepsilon_{r}}+\varepsilon_{r} \log \psi_{k}\right]-\phi$ has a local minimum at $\left[x^{\varepsilon_{r}}, t^{\varepsilon_{r}}\right]$,

and

$$
\left[x^{\varepsilon_{r}}, t^{\varepsilon_{r}}\right] \rightarrow\left(x_{0}, t_{0}\right) \text { as } r \rightarrow \infty \text {. }
$$

17 
Applying then the maximum princlple, we obtain from (2.6) ${ }_{c}$ the estimate

$$
0 \leq \phi_{t}-\varepsilon_{r} d_{k} \Delta \phi+d_{k}|D \phi|^{2}+\frac{f_{k}\left[u^{\varepsilon_{r}}\right]}{u_{k}^{\varepsilon_{r}}}
$$

$$
s \phi_{t}-\varepsilon_{r} d_{k} \Delta \phi+d_{k}|D \phi|^{2}+c_{k]} \exp \frac{\left[v_{k}^{\varepsilon_{r}}-v_{1}^{\varepsilon_{r}}\right]}{\varepsilon_{r}}
$$

at the point $\left[\mathrm{x}^{\varepsilon_{\mathrm{r}}}, \mathrm{t}^{\varepsilon_{\mathrm{r}}}\right]$, the second inequality being a consequence of hypothesis (F5). Using (3.9) and (3.11), we simplify (3.12) to read

$$
0 \leq \phi_{t}+d_{k}|D \phi|^{2}+c_{k 1} \frac{\psi_{1}}{\psi_{k}}+o(1) \text { as } \varepsilon_{r} \rightarrow 0
$$

at $\left(x_{0}, t_{0}\right)$. Since

$$
d_{k}|D \phi|^{2} \psi_{k}+c_{k l} \psi_{1}=\left(A\left(D \phi\left(x_{0}, t_{0}\right)\right) \psi_{k}=H\left(D \phi\left(x_{0}, t_{0}\right)\right) \psi_{k}\right.
$$

we deduce (3.7) upon letting $\varepsilon_{r} \rightarrow 0$.

3. We next verify assertion (11). To do so, we $f$ ix $\mu>0$ and select $\zeta \in C^{\infty}\left(\mathbb{R}^{n}\right)$ satisfying

$$
\left\{\begin{array}{l}
\zeta=0 \text { on } \bar{G}_{0}, \zeta>0 \text { on } R^{n}-\bar{G}_{0} \\
0 \leq \zeta \leq 1 .
\end{array}\right.
$$

We now claim that

$$
\max \left(v_{*}+H\left(D v_{*}\right), v_{*}-\mu \zeta\right) \geq 0 \text { on } R^{n} \times\{0\}
$$

In the viscosity sense, which means that if $\phi$ is a smooth test function and

$$
\left\{\begin{array}{l}
v_{*}-\phi \text { has a strict local minimum } \\
\text { at some point }\left(x_{0}, 0\right) \in R^{n} \times\{0\}
\end{array}\right.
$$

then elther 
(3. 15)

$$
v_{0}\left(x_{0}, 0\right) \geq \mu \zeta\left(x_{0}\right)
$$

or else

(3.16)

$$
\phi_{t}+H(D \phi) \geq 0 \text { at }\left(x_{0}, 0\right)
$$

Now if $x_{0} \in \bar{G}_{0}$, then (3.15) is clearly true. Otherwise suppose $x_{0} \in R^{n}-\bar{G}_{0}$ and

$$
v_{*}\left(x_{0}, 0\right)<\mu \zeta\left(x_{0}\right)<\infty \text {. }
$$

We repeat now the argument from Steps 1-2, noting in particular that since

$$
v_{k}^{\varepsilon_{r}}(x, 0)=+\infty \quad \text { for all } x \text { near } x_{0} \text {. }
$$

the points $\left[x^{\varepsilon_{r}}, t^{\varepsilon_{r}}\right]$ above lie in $\mathbb{R}^{n} \times(0, \infty)$. As such the maximum principle argument leading to inequality (3.12) is valid, and the rest of the proof proceeds as before, yielding at last the inequality (3.16).

4. Since

$$
v_{k}^{\varepsilon}(x, 0) \rightarrow 0 \quad \text { as } \varepsilon \rightarrow 0 \quad(k=1, \cdots, m)
$$

if $x_{0} \in G_{0}$, we have

$$
v_{*}=0 \text { on } G_{0} \times\{0\}
$$

To see that

$$
v_{*}=+\infty \text { on }\left(R^{n}-\bar{G}_{0}\right) \times\{0\}
$$

choose any point $x_{0} \in R^{n}-\bar{G}_{0}$ and suppose instead

$$
v_{n}(x, 0)<\infty \text {. }
$$

Fix $\delta>0$ and then define

$$
\phi^{\delta}(x, t)=-\frac{\left|x-x_{0}\right|^{2}}{\delta}-\lambda t,
$$

for $\lambda=\lambda(\delta)$ to be selected below. Since $v_{*}$ is lower semicontinuous, 


$$
v_{*}-\phi^{\delta} \text { has a minimum at a point }\left(x_{\delta}, t_{\delta}\right) \in R^{n} \times[0, \infty) \text {. }
$$

Then

$$
\frac{\left|x_{\delta}-x_{0}\right|^{2}}{\delta} \leq v_{n}\left(x_{\delta}, t_{\delta}\right)+\frac{\left|x_{\delta}-x_{0}\right|^{2}}{\delta}+\lambda t_{\delta} \leq v\left(x_{0}, 0\right)<\infty .
$$

Now if $t_{\delta}>0$,

$$
\phi_{t}^{\delta}+H(D \phi) \geq 0 \text { at }\left(x_{\delta}, t_{\delta}\right)
$$

whence

$$
-\lambda+H\left(-\frac{2\left(x_{\delta}-x_{0}\right)}{\delta}\right) \geq 0
$$

a contraction for $\lambda=\lambda(\delta)$ sufficiently large. Thus $t_{\delta}=0$. If

$$
v_{*}\left(x_{0}, 0\right)<\mu \zeta\left(x_{0}\right)
$$

then (3.19) implies

$$
v_{*}\left(x_{\delta}, 0\right)<\mu \zeta\left(x_{\delta}\right)
$$

for small enough $\delta$, and so according to (3.13) we once more would obtain (3.20). Thus

$$
v_{*}\left(x_{0}, 0\right) \geq \mu \zeta\left(x_{0}\right)
$$

But since $\zeta\left(x_{0}\right)>0$ and $\mu>0$ is arbitrary, (3.17) cannot be true.

Following next is the analogue of Proposition 3.1, with $v^{*}$ in place of $\mathbf{v}_{*}$

Proposition 3.2. We have

(1)

$$
\min \left(v_{t}^{*}+H(D v), v^{*}\right) \leq 0 \text { in } R^{n} \times(0, \infty)
$$

in the viscosity sense, and 
(1i)

(3.22)

$$
v=\left\{\begin{array}{lll}
0 & \text { on } & G_{0} \times\{0\} \\
+\infty & \text { on } & \left(R^{n}-\bar{G}_{0}\right) \times\{0\} .
\end{array}\right.
$$

Proof 1. Since $v * 0$, to establish (3.21) we must show

$$
v_{t}^{*}+H\left(D v^{*}\right) \leq 0 \text { on the set }\left\{v^{*}>0\right\} \text {. }
$$

in the viscosity sense. So select any smooth test function $\phi$ and suppose

(3.23) $\left\{\begin{array}{l}v-\phi \text { has a strict local maximum at some point } \\ \left(x_{0}, t_{0}\right) \in \mathbb{R}^{n} \times(0, \infty)\end{array}\right.$

with

$$
v\left(x_{0}, t_{0}\right)>0
$$

We need to show

$$
\phi_{t}+H(D \phi) \leq 0 \text { at }\left(x_{0}, t_{0}\right)
$$

Let $\left(\psi_{1}, \cdots, \psi_{k}\right)$ be a positive elgenvector of $A\left(D \phi\left(x_{0}, t_{0}\right)\right)$, corresponding to the principal elgenvalue $\lambda^{0}\left(A\left(D \phi\left(x_{0}, t_{0}\right)\right)=H\left(D \phi\left(x_{0}, t_{0}\right)\right)\right.$. Then

$$
v^{*}(x, t)=\underset{\substack{\varepsilon \rightarrow 0 \\(y, s) \rightarrow(x, t)}}{1 \max _{1 \leq 1 \leq m}}\left[v_{1}^{\varepsilon}(y, s)+\varepsilon \log \psi_{1}\right) .
$$

Combining (3.23) and (3.26), we deduce that there exists an index $k \in\{1, \cdots, m\}$, a sequence $\varepsilon_{r} \rightarrow 0$, and points $\left(x^{\varepsilon_{r}}, t^{\varepsilon_{r}}\right)$ such that

(3.27) $v_{k}^{\varepsilon_{r}}\left[x^{\varepsilon_{r}}, t^{\varepsilon_{r}}\right]+\varepsilon_{r} \log \psi_{k}=\max _{1 \leq 15 \mathrm{~m}}\left[v_{1}^{\varepsilon_{r}}\left[x^{\varepsilon_{r}}, t^{\varepsilon_{r}}\right]+\varepsilon_{r} \log \psi_{1}\right] \rightarrow v^{*}\left(x_{0}, t_{0}\right)$, (3.28) $v_{k}^{\varepsilon_{r}}\left[x^{\varepsilon_{r}}, t^{\varepsilon_{r}}\right)+\varepsilon_{r} \log \psi_{k}-\phi$ has a local maximum at $\left[x^{\varepsilon_{r}}, t^{\varepsilon_{r}}\right]$. 
and

$$
\left[x^{\varepsilon_{r}}, t^{\varepsilon_{r}}\right] \rightarrow\left(x_{0}, t_{0}\right) \text { as } r \rightarrow \infty
$$

Utilizing the maximum principle, we deduce from $(2.6)_{\varepsilon}$ the Inequality

$$
\phi_{t}-\varepsilon_{r} d_{k} \Delta \phi+d_{k}\left|D_{\phi}\right|^{\varepsilon} s-\frac{f_{k}\left[u^{\varepsilon_{r}}\right]}{u_{k}^{\varepsilon_{r}}}
$$

at the point $\left[x^{\varepsilon_{r}}, t^{\varepsilon_{r}}\right]$. We must now study the limiting behavior of the term on the right hand side as $r \rightarrow \infty$.

2. We assert

$$
u_{1}^{\varepsilon_{r}}\left[x^{\varepsilon_{r}}, \varepsilon^{\varepsilon_{r}}\right] \rightarrow 0 \quad(1=1, \cdots, m)
$$

as $r \rightarrow \infty$. To see this, note that

$$
u_{k}^{\varepsilon_{r}}\left[x^{\varepsilon_{r}}, t^{\varepsilon_{r}}\right]=\exp \left[-\frac{v_{k}^{\varepsilon_{r}}\left[x^{\varepsilon_{r}}, t^{\varepsilon_{r}}\right]}{\varepsilon_{r}}\right] \rightarrow 0,
$$

owing to (3.24) and (3.27). This establishes (3.31) for $1=k$. Now suppose additionally that for some index $1 \neq k$,

$$
\underset{r \rightarrow \infty}{\lim \sup } u_{1}^{\varepsilon_{r}}\left[x^{\varepsilon_{r}}, t^{\varepsilon_{r}}\right]=a>0
$$

Then passing if necessary through an appropriate subsequence $\{\varepsilon s\}_{s=1}^{\infty} \subset\left\{\varepsilon_{r}\right\}_{r=1}^{\infty}$, we find

$$
f_{k}\left[u^{\varepsilon_{u}}\left(x^{\varepsilon_{m}}, t^{\varepsilon_{z}}\right)\right] \rightarrow f_{k}(\cdots, a, \cdots, 0, \cdots,
$$

a > 0 occupying the $1^{\text {th }}$ argument and 0 occupying the $k^{\text {th }}$ argument of $f_{k}$. 
Using hypothesis (F2), we obtain

$$
\lim _{s \rightarrow \infty} \frac{f_{k}\left[u^{\varepsilon_{s}}\left(x^{\varepsilon_{s}}, t^{\varepsilon_{m}}\right]\right)}{u_{k}^{\varepsilon_{s}}\left(x^{\varepsilon_{s}}, t^{\varepsilon_{m}}\right)}=+\infty,
$$

a conclusion at varlance with (3.30). Thus statement (3.31) is true.

And Indeed the exact reasoning above proves additionally

$$
\frac{f_{k}\left[u^{\varepsilon_{r}}\right]}{u_{k}^{\varepsilon_{r}}}=O(1)
$$

as $r \rightarrow \infty$, the functions evaluated at the point $\left[x^{\varepsilon_{r}}, t^{\varepsilon_{r}}\right]$. We further refine this observation by claiming

$$
\frac{f_{k}\left[u^{\varepsilon_{r}}\right]}{u_{k}^{\varepsilon_{r}}}=\frac{c_{k l} u_{1}^{\varepsilon_{r}}}{u_{k}^{\varepsilon_{r}}}+o(1)
$$

as $r \rightarrow \infty$. To see this we observe that

$$
\begin{aligned}
f_{k}(u) & =f_{k}(0)+c_{k l} u_{1}+o\left(|u|^{2}\right) \\
& =c_{k l} u_{1}+o\left(|u|^{2}\right) \quad\left(u \in \mathbb{R}^{m}\right) .
\end{aligned}
$$

Now (3.31) yields

$$
\frac{\left|u^{\varepsilon_{r}}\right|^{2}}{u_{k}^{\varepsilon_{r}}}=o(1) \sum_{1=1}^{m} \frac{u_{1}^{\varepsilon_{r}}}{u_{k}^{\varepsilon_{r}}} \leq o(1) \frac{c_{k l} u_{l}^{\varepsilon_{r}}}{u_{k}^{\varepsilon_{r}}}
$$

as $r \rightarrow \infty$. Consequently (3.34) implies

$$
\frac{f_{k}\left[u^{\varepsilon_{r}}\right]}{u_{k}^{\varepsilon_{r}}} \geq(1-o(1)) \frac{c_{k l} u_{l}^{\varepsilon_{r}}}{u_{k}^{\varepsilon_{r}}}
$$

as $r \rightarrow \infty$. In view of (3.32) then 


$$
\frac{c_{k 1} u_{1}^{\varepsilon_{r}}}{u_{k}^{\varepsilon_{r}}}=O(1)
$$

as $\Gamma \rightarrow \infty$. Since

$$
\frac{f_{k}\left[u^{\varepsilon_{r}}\right]}{u_{k}^{\varepsilon_{r}}} \leq \frac{c_{k l} u_{1}^{\varepsilon_{r}}}{u_{k}^{\varepsilon_{r}}}
$$

according to hypothesis (F5), clalm (3.33) is proved.

Finally note that $(3.35) 1 \mathrm{mpl}$ les

$$
\frac{u_{1}^{\varepsilon_{r}}}{u_{k}^{\varepsilon_{r}}}=0(1) \quad(1=1, \cdots, m)
$$

as $r \rightarrow \infty$.

3. Owing to (3.27) and (3.33), inequality (3.30) ylelds

$$
\phi_{t}+d_{k}|D \phi|^{\varepsilon}+c_{k l} \frac{\psi_{1}}{\psi_{k}} \leq O(1) \text { as } \varepsilon_{r} \rightarrow \infty
$$

at the point $\left(x_{0}, t_{0}\right)$. We now conclude as in the proof of Proposition 3.1(1)

4. Next we verify assertion (11). We first claim

$$
\min \left(v_{t}^{*}+H\left(D v^{*}\right), v^{*}\right) \leq 0 \text { on } G_{0} \times\{0\}
$$

in the viscosity sense, whlch means that if $\phi$ is a smooth test function and

$$
\left\{\begin{array}{l}
v^{*}-\phi \text { has a strict local maximum at some point } \\
\left(x_{0}, 0\right) \in G_{0} \times\{0\}
\end{array}\right.
$$

then el ther

(3. 38)

$$
v\left(x_{0}, 0\right)=0
$$

or else

(3. 39)

$$
\phi_{t}+H(D \phi) \leq 0 \text { at }\left(x_{0}, 0\right)
$$


Now if (3.38) is false, then we repeat the argument from Steps 1-3, above noting that since

$$
v_{k}^{\varepsilon}(x, 0)=0 \text { for all } x \text { near } x_{0} \text {. }
$$

the points $\left[x^{\varepsilon_{r}}, t^{\varepsilon_{r}}\right]$ lie in $R^{n} \times(0, \infty)$. Consequently the maximuin principle arguments employed above lead us to (3.39).

5. Now observe that

$$
v^{*}=+\infty \quad \text { on }\left(\mathbb{R}^{n}-\bar{G}_{0}\right) \times\{0\}
$$

since $v_{k}^{\varepsilon}=+\infty$ on that set. Suppose then that $x_{0} \in G_{0}$, but

$$
v\left(x_{0}, 0\right)>0
$$

Fix $\delta>0$ and write

$$
\phi^{\delta} \equiv \frac{\left|x-x_{0}\right|^{2}}{\delta}+\lambda t
$$

for $\lambda=\lambda(\delta)$ to be chosen. Since $v$ is upper semicontinuous and is bounded near $x_{0}$,

$$
\left\{\begin{array}{l}
v^{*}-\phi^{\delta} \text { has a local maximum at a point } \\
\left(x_{\delta}, t_{\delta}\right) \in G_{0} \times(0, \infty)
\end{array}\right.
$$

for each sufficiently small $\delta>0$, with

$$
x_{\delta} \rightarrow x_{0} \text { as } \delta \rightarrow 0
$$

If $t_{\delta}>0$, then

$$
\phi_{t}^{\delta}+H\left(D \phi^{\delta}\right) \leq 0 \text { at }\left(x_{\delta}, t_{\delta}\right)
$$

whence

$$
\lambda+H\left(\frac{2\left[x_{\delta}-x_{0}\right]}{\delta}\right] \leq 0,
$$


a contradiction for $\lambda=\lambda(\delta)$ large enough. Thus $t_{\delta}=0$.

Now

$$
0<v\left(x_{0}, 0\right] \leq v *\left(x_{\delta}, 0\right)-\frac{\left|x_{\delta}-x_{0}\right|^{2}}{\delta} ;
$$

owing to (3.37) we once again reach the contradiction (3.42). Hence (3.41) is untenable, so that

$$
v^{*}=0 \text { on } G_{0} \text {. }
$$

as required.

$\square$

\section{Concluston of the proof of Theorem 1.}

In light of Propositions 3.1 and 3.2 we may invoke the uniqueness theorems developed in the Appendix, $\S 4$, to find

$$
v^{*}=v_{*}=I \quad \text { in } R^{n} \times(0, \infty) \text {. }
$$

where I is the unique viscosity solution of the Hamilton-Jacobl varlational inequality

$$
\begin{cases}\min \left(I_{t}+H(D I), I\right)=0 & \text { in } \mathbb{R}^{n} \times(0, \infty) \\ I=0 & \text { on } G_{0} \times\{0\} \\ I=+\infty & \text { on }\left(\mathbb{R}^{n}-\bar{G}_{0}\right) \times\{0\} .\end{cases}
$$

Additionally,

(3.44) $\quad v_{k}^{\varepsilon} \rightarrow I$ uniformly on compact subsets of $R^{n} \times(0, \infty), \quad k=1, \cdots, m$.

Now according to $\$ 5$ in [6], we have

$$
I=\max (\mathrm{J}, 0) \text {, }
$$

where $J$ is the unique viscosity solution of the Hamilton-Jacobl equation 


$$
\begin{cases}J_{t}+H(D J)=0 & \text { in } R^{n} \times(0, \infty) \\ J=0 & \text { on } G_{0} \times\{0\} \\ J=+\infty & \text { on }\left(R^{n}-\bar{G}_{0}\right) \times\{0\} .\end{cases}
$$

In addition we have the representation formula

$$
J(x, t) \equiv \inf \left\{\int_{0}^{t} L(\dot{z}(s)) d s \mid z(0) \in G_{0}, z(t)=x\right\}
$$

the infimum taken over all absolutely cont Inuous functions

$$
z:[0, t] \rightarrow \mathbb{R}^{n}
$$

Now since (2.5) and (3.44) $1 \mathrm{mply}$

$$
u_{k}^{\varepsilon}=e^{-\frac{v_{k}^{\varepsilon}}{\varepsilon}}=e^{-\frac{I+o(1)}{\varepsilon}} \quad(k=1, \cdots, m),
$$

we see that

(3.48) $\quad u_{k}^{\varepsilon} \rightarrow 0$ uniformly on compact subsets of $\{I>0\}=\{J>0\}$

as $\varepsilon \rightarrow 0$, for $k=1, \cdots, m$.

We must now show

(3.49) $\quad \underset{\varepsilon \rightarrow 0}{\lim \inf } \min _{1 \leq k \leq m} u_{k}^{\varepsilon}>0$ uniformly on compact subset of $\{J<0\}$.

So fix any point $\left(x_{0}, t_{0}\right) \in\{J<0\}$. Then $I=0$ near $\left(x_{0}, t_{0}\right)$. Define

$$
\phi(x, t)=\left|x-x_{0}\right|^{2}+\left|t-t_{0}\right|^{2}
$$

Owing to (3.44) we see that for each $k=1, \cdots, m$.

$$
v_{k}^{\varepsilon}-\phi \text { has a maximum at a point }\left(x_{k}^{\varepsilon}, t_{k}^{\varepsilon}\right] \text {, }
$$

with

(3.51)

$$
\left[x_{k}^{\varepsilon}, t_{k}^{\varepsilon}\right] \rightarrow\left(x_{0}, t_{0}\right) \quad \text { as } \varepsilon \rightarrow 0
$$


Applying the maximum principle, we find using (2.6) $)_{\varepsilon}$ that

$$
o(1)=\phi_{t}-\varepsilon d_{k} \Delta \phi+d_{k}|D \phi|^{2} \leq-\frac{f_{k}\left[u^{\varepsilon}\right]}{u_{k}^{\varepsilon}} \text {; }
$$

and so

$$
f_{k}\left(u^{\varepsilon}\right) \leq o(1) u_{k}^{\varepsilon} \text { at the point }\left[x_{k}^{\varepsilon}, t_{k}^{\varepsilon}\right] \text {. }
$$

as $\varepsilon \rightarrow 0$.

Now there exists a constant $\alpha>0$ such that

$$
f^{k}(u) \geq c_{k 1} u_{1}-\alpha|u|^{2} \quad \text { for all } u \in \mathbb{R}^{m} \text {. }
$$

Let us suppose first that

$$
u_{1}^{\varepsilon}\left[x_{k}^{\varepsilon}, t_{k}^{\varepsilon}\right] \leq \frac{c_{k l}}{2 \alpha} \quad \text { for each } \quad 1=1, \cdots, m, 1 \neq k \text {. }
$$

Then from (3.53) and (3.54) we deduce

$$
o(1) u_{k}^{\varepsilon} \geq f_{k}\left(u^{\varepsilon}\right) \geq \sum_{l \neq k} c_{k l} u_{l}^{\varepsilon}+c_{k k} u_{k}^{\varepsilon}-\alpha\left|u^{\varepsilon}\right|^{2}
$$

at $\left[x_{k}^{\varepsilon}, t_{k}^{\varepsilon}\right] ;$ whence (3.55) implies

$$
u_{k}^{\varepsilon}\left[x_{k}^{\varepsilon}, t_{k}^{\varepsilon}\right] \geq \frac{c_{k k}}{2 \alpha}
$$

for $\varepsilon$ small enough. Should (3.55) fall, then

$$
u_{1}^{\varepsilon}\left[x_{k}^{\varepsilon}, t_{k}^{\varepsilon}\right]>\frac{c_{k 1}}{2 \alpha} \text { for some } 1 \neq k \text {. }
$$

But then owing to hypothesis (F2)

$$
f^{k}\left(\cdots u_{k-1}^{\varepsilon}, 0, u_{k+1}^{\varepsilon}, \cdots\right) \geq \beta
$$

at the point $\left[x_{k}^{\varepsilon}, t_{k}^{\varepsilon}\right]$, for some positive constant $\beta$. Thus at $\left[x_{k}^{\varepsilon}, t_{k}^{\varepsilon}\right]$ we 
have

$$
\begin{gathered}
f^{k}\left(u^{\varepsilon}\right) \geq \beta+f^{k}\left(u^{\varepsilon}\right)-f^{k}\left(\cdots u_{k-1}^{\varepsilon}, 0, u_{k+1}^{\varepsilon} \cdots\right) \\
\geq \beta+c_{k k} u_{k}^{\varepsilon}-\gamma\left[\left[u_{k}^{\varepsilon}\right]^{2}+\sum_{1=1}^{m} u_{k}^{\varepsilon} u_{1}^{\varepsilon}\right]
\end{gathered}
$$

for some $\gamma>0$. Consequently (3.53) Implies

$$
\left(u_{k}^{\varepsilon}\right)^{2} \geq \beta+o\left(\left|u_{k}^{\varepsilon}\right|\right) \text { at }\left[x_{k}^{\varepsilon}, t_{k}^{\varepsilon}\right] \text {. }
$$

and so

$$
u_{k}^{\varepsilon}\left[x_{k}^{\varepsilon}, t_{k}^{\varepsilon}\right] \geq \delta>0
$$

for sufficiently small $\delta>0$. But since

$$
\left[v_{k}^{\varepsilon}-\phi\right]\left[x_{k}^{\varepsilon}, t_{k}^{\varepsilon}\right] \geq\left[v_{k}^{\varepsilon}-\phi\right]\left[x_{0}, t_{0}\right]
$$

we have

$$
v_{k}^{\varepsilon}\left[x_{k}^{\varepsilon}, t_{k}^{\varepsilon}\right] \geq v_{k}^{\varepsilon}\left[x_{0}, t_{0}\right]
$$

and so

$$
u_{k}^{\varepsilon}\left[x_{k}^{\varepsilon}, t_{k}^{\varepsilon}\right] \leq u_{k}^{\varepsilon}\left[x_{0}, t_{0}\right]
$$

Thus

$$
\lim _{\varepsilon \rightarrow 0} \operatorname{lnf} u_{k}^{\varepsilon}\left[x_{0}, t_{0}\right] \geq \delta>0 .
$$

\section{Appendix: Identification of the action function}

We outline in this section a proof that the functions $v_{\text {. }}$ and $v$ introduced in 53 agree and equal $I$, the unlque viscosity solution of

(4.1)

$$
\begin{cases}\min \left(I_{t}+H(D I), I\right)=0 & \text { in } R^{n} \times(0, \infty) \\ I=0 & \text { on } G_{0} \times\{0\} \\ I=+\infty & \text { on }\left(R^{n}-\bar{C}_{0}\right) \times\{0\} .\end{cases}
$$


First we show

$$
v . \geq I \quad \text { in } R^{n} \times(0, \infty) \text {. }
$$

For this choose any constant $\mu>0$ and any function $\zeta \in C^{\infty}\left(R^{n}\right)$ satisfying

$$
\left\{\begin{array}{l}
\zeta \equiv 0 \text { on } \bar{G}_{0}, \zeta>0 \text { on } \mathbb{R}^{n}-\bar{G}_{0} \\
0 \leq \zeta \leq 1
\end{array}\right.
$$

Consider now the auxiliary problem

$$
\begin{cases}\min \left(I_{\mu \mathrm{t}}+\mathrm{H}\left(\mathrm{DI}{ }_{\mu}\right), I_{\mu}\right)=0 & \text { in } \mathbb{R}^{\mathrm{n}} \times(0, \infty) \\ I_{\mu}=\mu \zeta & \text { on } \mathbb{R}^{\mathrm{n}} \times(0, \infty) .\end{cases}
$$

which has a unique, continuous solution according to [6]. Furthermore, according to assertion (11) in Proposition 3.1 we have

$$
v_{*} \geq I_{\mu}
$$$$
\text { on } \mathbb{R}^{\mathrm{n}} \times\{0\} \text {. }
$$

Finally, $v_{*}$ is lower semicontinuous, and

$$
\min \left(v_{*}+H\left(D v_{*}\right), v_{*}\right) \geq 0 \quad \text { in } \mathbb{R}^{n} \times(0, \infty)
$$

in the viscosity sense. Consequently a comparison argument

following [3] and [6] Implies

$$
v_{*} \geq I_{\mu}
$$$$
\text { in } R^{n} \times(0, \infty)
$$

Letting $\mu$ tend to infinity we have

$$
I_{\mu} \rightarrow I \quad \text { in } R^{n} \times(0, \infty) \text {; }
$$

and so (4.2) follows.

Next we assert that

$$
v \leq I
$$

In $R^{n} \times(0, \infty)$. 
To prove this define for each small $\delta>0$ the smooth set

$$
G_{\delta}=\left\{x_{0} \in G_{0} \mid d i s t\left(x, R^{n}-G_{0}\right)>\delta\right\} .
$$

Fix $\delta>0$ and write

$$
\Lambda_{\sigma}=\sup _{x \in G_{\delta}} v(x, \sigma) \quad(\sigma>0) .
$$

Since $v$ is upper semicont inuous and

$$
v=0 \text { on } G_{0} \times\{0\} \text {. }
$$

according to assertion (11) of Proposition 3.2, we see that

$$
\lim _{\sigma \rightarrow 0} \Lambda_{\sigma}=0
$$

for each fixed $\delta>0$. Choose some small $\sigma>0$ and consider the problem

$$
\begin{cases}\min \left[I_{t}^{\delta, \sigma}+H(D I, \sigma), I^{\delta, \sigma}\right)=0 & \text { in } \mathbb{R}^{\mathrm{n}} \times(\sigma, \infty) \\
I^{\delta, \sigma}=\left\{\begin{array}{cl}
\Lambda_{\delta} & \text { on } G_{\delta} \times\{\sigma\} \\
+\infty & \text { on }\left(\mathbb{R}^{n}-\bar{G}_{\delta}\right) \times\{\sigma\}
\end{array} .\right.\end{cases}
$$

In view of Proposition 3.2 we have

$$
v^{*} \leq I^{\delta, \sigma} \quad \text { on } R^{n} \times\{\sigma\}
$$

Since additionally $v$ is upper semicontinuous and

$$
\operatorname{mln}\left(v_{t}^{*}+H\left(D v^{*}\right), v^{*}\right) \leq 0 \quad \text { in } R^{n} \times(\sigma, \infty)
$$

in the viscosity sense, we have

$$
v^{*} \leq I^{\delta, \sigma} \quad \text { in } R^{n} \times(\sigma, \infty) .
$$

Let $\sigma \rightarrow 0$ and recall (4.7) to discover

$$
v \leq I^{\delta}
$$

on $R^{n} \times(0, \infty)$,

when $I^{\delta}$ is the unlque viscosity solution of 


$$
\begin{cases}m \ln \left[I_{t}^{\delta}+H\left(D I^{\delta}\right), I^{\delta}\right)=0 & \text { in } R^{n} \times(0, \infty) \\
I^{\delta}=\left\{\begin{array}{cl}
0 & \text { on } G_{\delta} \times\{0\} \\
+\infty & \text { on }\left(R^{n}-\bar{G}_{\delta}\right) \times\{0\}
\end{array} .\right.\end{cases}
$$

Now at last send $\delta \rightarrow 0:$ since

$$
I^{\delta} \rightarrow I \quad \text { in } R^{n} \times(0, \infty)
$$

we arrive at (4.5).

Combining (4.2) and (4.5) we have

$$
v \leq I \leq v_{*} \quad \text { in } R^{n} \times(0, \infty) .
$$

But since the definitions imply obviously that

$$
v_{*} \leq v^{*} \quad \text { in } \mathbb{R}^{n} \times(0, \infty),
$$

we have

$$
v^{*}=v_{*}=I \quad \text { in } R^{n} \times(0, \infty)
$$

Another approach to obtain the above is to modify (in a more or less stralght forward way) the results of M.G. Crandall, P. -L. Lions and P.E. Souganidis [4] concerning maximal solutions. 


\section{References}

1. G. Barles and B. Perthame, Exit time problems in optimal control and vanishing viscosity method, to appear.

2. M.G. Crandall, L.C. Evans, and P.L. Llons. Some propertles of viscosity solutions of Hamilton-Jacobl equations, Trans. AMS 282(1984), 487-502.

3. M.G. Crandall and P.L. Llons, Viscosity solutions of Hamllton-Jacobl equations, Trans. AMS 277 (1983), 1-42.

4. M.G. Crandall, P.L. Lions, and P.E. Souganidls. Universal bounds and maximal solutions for certain evolution equations, to appear in Arch. Anal. Rat. Mech.

5. L.C. Evans and H. Ish11, A PDE approach to some asymptotic problems concerning random differentlal equations with small nolse intensities, Ann. L' Institut H. Poincare 2(1985), 1-20.

6. L.C. Evans and P.E. Souganidis, A PDE approach to geometric optics for certain semilinear parabolic equations, to appear in Indiana University Math. J.

7. L.C. Evans and P.E. Souganidis, A PDE approach to certain large deviation problems for systems of parabolic equations, to appear in Ann.

L'Institute H. Polncare.

8. W.H. Fleming, Logarithmic transformations and stochastic control, Advances in Filtering and Opt Imal Stochast ic Control, ed. by W.H. Fleming and L.G. Gorostiza, Springer-Verlag.

9. M. I. Freidlin, Funct lonal Integrat ion and Partial Differential Equations, Annals of Math. Studies 109, Princeton Unlversity Press, Princeton, 1985.

10. M.I. Freidlin, Limit theorems for large deviations and reaction-diffusion equations, Annals of Prob. 13(1985), 639-675.

11. H. Ish11, A boundary value problem of Dirichlet type for Hamilton-Jacob1 equat ions. 